1976

\title{
Homogeneous nucleation in associated vapors. I. Acetic acid
}

\author{
Richard H. Heist \\ Fairfield University, rheist@fairfield.edu
}

Kevin M. Colling

Calvin S. DuPuis

Follow this and additional works at: https://digitalcommons.fairfield.edu/engineering-facultypubs (C) 1976 American Institute of Physics.

The final publisher PDF has been archived here with permission from the copyright holder. https://aip.scitation.org/doi/abs/10.1063/1.433056

\section{Peer Reviewed}

\section{Repository Citation}

Heist, Richard H.; Colling, Kevin M.; and DuPuis, Calvin S., "Homogeneous nucleation in associated vapors. I. Acetic acid" (1976). Engineering Faculty Publications. 160.

https://digitalcommons.fairfield.edu/engineering-facultypubs/160

\section{Published Citation}

Heist, R. H., Colling, K. M., \& DuPuis, C. S. (1976). Homogeneous nucleation in associated vapors. I. Acetic acid. The Journal of Chemical Physics, 65(12), 5147-5154. doi:10.1063/1.433056.

This item has been accepted for inclusion in DigitalCommons@Fairfield by an authorized administrator of DigitalCommons@Fairfield. It is brought to you by DigitalCommons@Fairfield with permission from the rightsholder(s) and is protected by copyright and/or related rights. You are free to use this item in any way that is permitted by the copyright and related rights legislation that applies to your use. For other uses, you need to obtain permission from the rights-holder(s) directly, unless additional rights are indicated by a Creative Commons license in the record and/or on the work itself. For more information, please contact digitalcommons@fairfield.edu. 


\title{
Homogeneous nucleation in associated vapors. I. Acetic acid
}

\author{
Richard H. Heist, ${ }^{*}$ Kevin M. Colling, ${ }^{\dagger}$ and Calvin S. DuPuis \\ Interfacial Science Group, Department of Chemical Engineering, University of Rochester, Rochester, New York 14627
} (Received 10 August 1976)

\begin{abstract}
Homogeneous nucleation measurements on acetic acid vapor are reported. The presence of the relatively stable association clusters tends to stabilize the vapor with regard to homogeneous nucleation. The variation of the critical supersaturation with temperature for acetic acid vapor was found to agree well with the predictions of the Katz-Saltsburg-Reiss theory for nucleation in associated vapors.
\end{abstract}

\section{INTRODUCTION}

The theory of homogeneous nucleation developed by Volmer and Weber, ${ }^{1}$ and revised by Becker and Döring, ${ }^{2}$ and others ${ }^{3,4}$ applies only to vapors consisting of a single molecular species. This theory, commonly known as the conventional or Becker-Döring nucleation theory, neglects all effects due to the relatively stable clusters present in associated vapors. Although there have been attempts in the literature to revise the conventional theory by including these effects, virtually nothing has been done by way of experimental measurements. This dearth of experimental information is particularly disturbing when one considers that, of the two best known theories for nucleation in associated vapors, one predicts the completely opposite effect of the other. The purpose of this paper is to report new experimental results on the homogeneous nucleation of acetic acid vapor and to compare those results with existing theories for nucleation in associated vapors.

In 1954, Frisch and Willis ${ }^{5}$ considered a vapor composed of monomer and dimer and derived a nucleation rate expression corrected for the presence of the dimer. They reasoned that the role of dimer in the nucleation process was to provide an alternative path for the growth of a precritical size embryo. (For our discussion, we make a distinction between clusters present in an associated vapor and embryos of the same size normally considered as part of the nucleation size distribution. This is purely for ease in description. Both theories discussed here treat association clusters and embryos as indistinguishable.) The embryo can now change size by gaining or losing both monomer and dimer; consequently, the number of steps necessary to "grow" a critical size embryo is reduced. This is a purely kinetic agrument with the result being that dimer will destabilize a supersaturated vapor with respect to condensation. According to the FW (Frisch, Willis) theory, condensation in a vapor containing both monomer and dimer should occur at lower supersaturations than in the same vapor containing the monomer alone. They give as an approximate expression for the rate of nucleation in a monomer-dimer vapor, the equation

$$
J_{\mathrm{FW}}=J^{\prime}\left(1+2^{-1 / 2} B_{12}\right) \text {, }
$$

where $J_{\mathrm{FW}}$ is the rate of nucleation in drops $/ \mathrm{cm}^{3} \mathrm{sec}$, $B_{12}$ is the total surface fraction of dimer to monomer, and $J^{\prime}$ is the conventional nucleation rate expression

$$
J^{\prime}=\frac{a}{d}\left(\frac{2 N_{0}^{3} \sigma M}{\pi}\right)^{1 / 2}\left(\frac{P_{\theta} S}{R T}\right)^{2} \exp \left[-\frac{16 \pi N_{0}}{3(\ln S)^{2}}\left(\frac{M}{d}\right)^{2}\left(\frac{\sigma}{R T}\right)^{3}\right] .
$$

In Eq. (2), $a$ is the condensation coefficient, $d$ is the liquid density, $N_{0}$ is Avogadro's number, $\sigma$ is the bulk liquid surface tension, $M$ is the molecular weight, $P_{e}$ is the equilibrium vapor pressure, $S$ is the supersaturation ratio (defined as $P / P_{\theta}$ with $P$ being the existing pressure), and $R$ is the gas constant. It is important to note that the FW theory predicts a multiplicative, preexponential correction to $J^{\prime}$ and that the exponential term in the conventional nucleation rate expression remains unchanged.

In 1966, Katz, Saltsburg, and Reiss ${ }^{6}$ presented a somewhat more general theory for nucleation in associated vapors. Association clusters up to a size $m$ were considered with $m$ representing the number of monomers in the largest cluster present in the vapor. In this theory, as in the FW theory, association clusters and embryos of the same size were considered to be indistinguishable. The KSR (Katz, Saltsburg, Reiss) theory included the kinetic effect suggested earlier by Frisch and Willis; but, in addition, found that the presence of association clusters influences the embryo size distribution. The equation for the rate of nucleation in an associated vapor derived in the KSR theory is

$$
\begin{aligned}
J= & \frac{a}{d}\left(\frac{2 N_{0}^{3} \sigma M}{\pi}\right)^{1 / 2}\left(\frac{P_{e} S}{R T}\right)^{2} \\
& \times \sum_{i=1}^{m} i^{3 / 2} x_{i} \exp \left[-\frac{16 \pi N_{0}}{3\left(\ln S_{1}\right)^{2}}\left(\frac{M}{d}\right)^{2}\left(\frac{\sigma}{R T}\right)^{3}\right] .
\end{aligned}
$$

Here $J$ is the nucleation rate in drops $/ \mathrm{cm}^{3} \mathrm{sec}^{-1}, i$ is the number of molecules in an association cluster of size $i$, $x_{i}$ is the ratio $P_{i} / P$ (with $P_{i}$ being the partial pressure of the $i$-size clusters and $P$ the actual pressure of the vapor), $S$ is the supersaturation ratio $P / P_{e}$ (where $P_{e}$ is the equilibrium vapor pressure), and $S_{1}$ is the monomer supersaturation ratio $P_{1} / P_{1 e}$ (where $P_{1 e}$ is the equilibrium vapor pressure of the monomer only). The remaining symbols have the same meaning as in Eq. (2). The correction for the kinetic effect occurs, as in the FW theory, in the pre-exponential term; but the effect of association on the embryo distribution shows up in the exponential term. The monomer supersaturation ratio, $S_{1}$, has replaced the total supersaturation ratio, $S$, in the exponential in Eq. (3). In associated vapors $S_{1}$ will be less than $S$ resulting in an increase in the total super- 


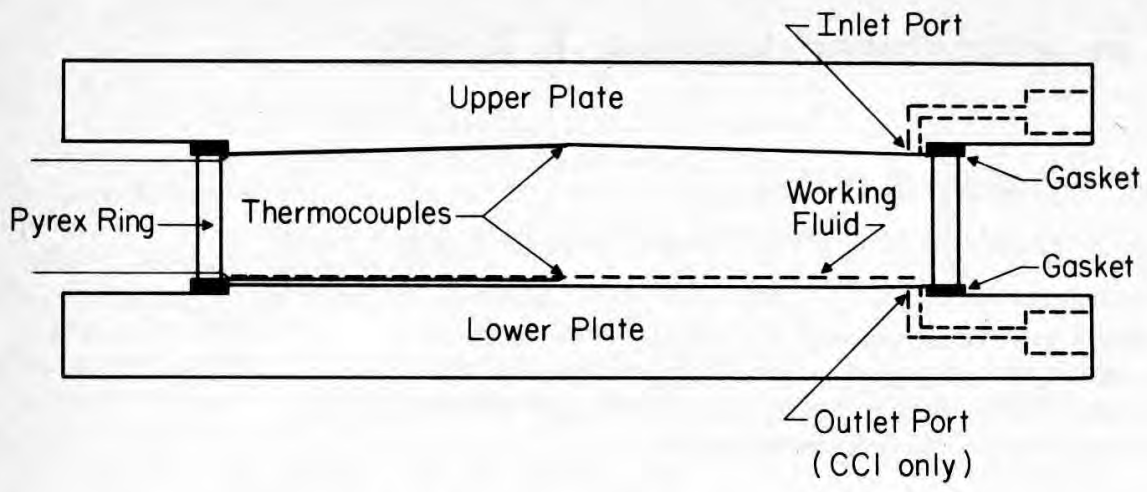

FIG. 1. A general schematic for both diffusion cloud chambers used in this investigation. Three nichrome heater wires around the Pyrex ring have been omitted as have the individual plate temperature baths. See text for discussion. The schematic is not drawn to scale.

saturation necessary for condensation. This thermodynamic effect tends to stabilize the supersaturated vapor with respect to condensation and completely dominates the relatively minor kinetic effect. According to the KSR theory a vapor containing both monomer and dimer should require larger supersaturations for condensation to occur than if the vapor contained monomer only.

As late as 1971 in a paper by Kiang et al. ${ }^{7}$ reviewing various aspects of nucleation theory, the effects of vapor phase association on the nucleation rate were discussed and the authors came to a conclusion similar to Frisch and Willis. Kiang et $a l$. allowed for the kinetic effect as did the FW and KSR theories but found that in their particular treatment the larger thermodynamic effect predicted in the KSR theory did not occur.

Two different versions of a diffusion cloud chamber were used in this investigation and both are discussed briefly in the following section. The diffusion cloud chamber was chosen because its characteristic quiescent, steady-state operation permits chamber conditions to be well defined and maintained conveniently for long periods of time. Acetic acid was chosen as a working substance because it is known to be heavily associated in the vapor phase. ${ }^{8-11}$

\section{CHAMBER DESIGN AND OPERATION}

The two cloud chambers used for this work are based on designs described in exhausting detail in previous publications, ${ }^{12-15}$ so only important variations will be discussed in detail here.

Both chambers are represented by the schematic shown in Fig. 1. The first chamber, CC1, is comprised of two circular aluminum plates $2.8 \mathrm{~cm}$ thick and $30.5 \mathrm{~cm}$ in diameter. These plates are separated by a Pyrex ring $3.8 \mathrm{~cm}$ high and $0.9 \mathrm{~cm}$ thick with an inside diameter of $28.6 \mathrm{~cm}$ providing the actual working area. The Pyrex ring is separated from the two aluminum plates by ethylene-propylene gaskets (this composition is relatively immune to acetic acid attack). The upper plate surface has a $1^{\circ}$ bevel to allow condensed fluid to drain to the chamber wall. There are two ports, each $0.16 \mathrm{~cm}$ in diameter, in the upper plate to permit acetic acid to be added and for control of the chamber environment. There is a $0.16 \mathrm{~cm}$ diameter port in the lower plate to permit drainage. During op- eration the lower plate port is sealed by a Teflon covered plug. The lower (upper) plate is maintained at temperature by a hot (cold) bath assembly fastened directly to the plate. ${ }^{14}$ The temperature of the bath assembly is controlled by an external constant temperature circulation system. The temperature control for each plate is quite sensitive and changes of a few hundreths of a degree may be made with ease. During actual operation the plate temperatures remain constant and usually vary by less than $0.1{ }^{\circ} \mathrm{C}$ over most of the working surface. The temperature of each plate does vary near the Pyrex ring, however, because of edge effects. These are overcome by maintaining a diameter : height ratio $>5 .{ }^{12,13}$ The diameter : height ratio for CC1 is 7 .

In $\mathrm{CC} 1$ acetic acid is maintained as a shallow pool, approximately $0.1-0.2 \mathrm{~cm}$ deep, on the lower plate and as a thin film of condensate on the upper plate surface. The important temperatures in these experiments are the two liquid surface temperatures. These are measured directly by two thermocouples brought into the chamber through the Pyrex ring. This is a recent innovation ${ }^{16}$ and will be described in more detail. In CC1 the interior thermocouples are of 26 gauge chromelalumel wire. This composition has good corrosion resistance with respect to acetic acid. Both couples are fed into the chamber through two sets of holes drilled in the Pyrex ring, each hole being slightly larger than the wire diameter. The holes in each set are 2.54 $\mathrm{cm}$ apart and $0.64 \mathrm{~cm}$ from the edge of the Pyrex ring. The holes and the wires are sealed from the outside by a low vapor pressure epoxy cement (Torr Seal, Varian Assoc. ). Once through the Pyrex ring, the bottom thermocouple conforms to the ring surface until it reaches the pool; it is then positioned to extend just below the pool surface to the chamber center (see Fig. 1). The thermocouple junction is bent up slightly so it dimples the pool surface. The upper thermocouple, which is flat $(0.012 \mathrm{~cm}$ thick), conforms to the ring surface until it reaches the upper plate and is then positioned to rest flush against the upper plate surface and extend chamber center so the junction is over the lower surface junction (see Fig. 1). The reason for flat wire and insisting upon uniform contact along the upper plate surface is to insure that the liquid film covering the upper plate surface also covers the wire. This is in fact what actually happens.

During operation the upper and lower plates were electrically insulated from each other and electric 
fields up to $90 \mathrm{~V} / \mathrm{cm}$ were applied to sweep out ions resulting from cosmic ray or natural radioactivity sources. A small amount of wall heat, usually 4-8 W supplied from three heater wires wrapped about the Pyrex ring, was necessary to just free the inside of the Pyrex ring from condensation and permit visual access. A $650 \mathrm{~W}$ tungsten-halide lamp and a $2.0 \mathrm{~mW} \mathrm{He}-\mathrm{Ne}$ laser were used to observe the nucleation rate (not at the same time). The light from the tungsten-halide lamp was collimated and directed through the chamber. The light scattered from the falling droplets permitted the nucleation rate to be determined visually. The beam was used both with and without a heat filter with no noticeable difference. The laser beam was shaped into a rectangular beam approximately $1.5 \mathrm{~cm}$ wide by 0.1 $\mathrm{cm}$ thick and directed through the chamber approximately $1 \mathrm{~cm}$ from the lower surface. Although the entire trace of the falling droplet could not be observed using the laser, the scattered light was more intense and easily seen when the drop fell through the beam. Experimental results were the same with either light source.

The second chamber, CC2, differs in several respects from CC1. First of all, although constructed of aluminum, the upper and lower plates are only $13.9 \mathrm{~cm}$ in diameter and $1.75 \mathrm{~cm}$ thick. The Py rex glass ring separating the two plates is $2.54 \mathrm{~cm}$ high and $0.45 \mathrm{~cm}$ thick with i. d. $=14.4 \mathrm{~cm}$. The ring is separated from the plates by Teflon covered Viton gaskets. CC2 is considerably smaller than $\mathrm{CC} 1$, but the diameter : height ratio is 6.5 . Both of the chamber plates are coated with $0.025 \mathrm{~cm}$ of Teflon. There are two ports, each 0.16 $\mathrm{cm}$ in diameter, in the upper plate to add acetic acid and control the chamber atmosphere but no drain port in the lower plate. The only exposed aluminum surface in CC2 is the relatively small area inside the inlet ports (see Fig. 1). Since acetic acid does not wet Teflon, the upper plate must be covered with a thin layer of cleaned glass wool. This particular technique was first described elsewhere. ${ }^{14}$ The pool of acetic acid is maintained approximately $0.3 \mathrm{~cm}$ deep. Two thermocouples are brought in through the Pyrex wall and positioned in the manner described earlier except that in this case they are of $\mathbf{2 8}$ gauge copper-constantan wire, and the upper surface thermocouple wire is not flat. Instead, it is nested on the surface of the glass wool. Both junctions are aligned and extend to about $2 \mathrm{~cm}$ from chamber center.

The operating mechanics for CC2 are similar to CC1. The plates were heated in the same fashion, plate temperature behavior was nearly the same, electric fields up to $140 \mathrm{~V} / \mathrm{cm}$ were used to sweep out ions, wall heat (generally 5-10 W) was used to free the inside of the ring from condensation, and the $2.0 \mathrm{~mW} \mathrm{He-Ne} \mathrm{laser}$ was used to observe the nucleation rate as described earlier.

Both cloud chambers were tested extensively prior to the acetic acid experiments discussed in this paper. Homogeneous nucleation experiments were performed with water and ethanol in CC2 and $n$-octane in CC1 with all results agreeing with published data. ${ }^{12-14}$
During actual operation both chambers function similarly. In brief, the working fluid evaporates from the lower pool at a higher temperature and diffuses through a light, inert carrier gas to the upper plate where it condenses at a lower temperature forming a film which runs off to the edge and drains down the wall to the pool. The light carrier gas is necessary to maintain a decreasing density gradient from the lower to the upper plate thus preventing convection. When properly run the chamber operates in a quiescent, steady-state mode with very nearly plane parallel diffusion of the working fluid vapor. The actual pressure and temperature profiles within the chamber are obtained from the solutions to the appropriate one dimensional energy and mass transport equations. The equations are discussed in the following section. A more detailed description of chamber operation is given elsewhere. ${ }^{12-15}$

\section{THE TRANSPORT EQUATIONS}

Up to now, most nucleation experiments with the diffusion cloud chamber have involved only two component systems; a carrier gas and a working fluid vapor of a single molecular species. The transport of mass and energy between the chamber plates has been well characterized by solution of the mass and energy transport equations. ${ }^{15}$ The solutions to these equations yield the partial pressure and temperature profiles throughout the chamber necessary to calculate the vapor supersaturation.

There are two serious complications which arise when acetic acid is used as the working fluid; first, there are two (and perhaps more) molecular species present in the working fluid vapor, i. e., monomer and dimer; and, second, there is the chemical reaction

$$
2 \mathrm{CH}_{3} \mathrm{COOH}=\left(\mathrm{CH}_{3} \mathrm{COOH}\right)_{2}
$$

going on during the transport. We can, however, with certain simplifications derive approximate relations which will allow us to calculate the pressure and temperature profiles within the chamber.

In the treatment that follows we neglect all terms involving coefficients of thermal diffusion. The role of thermal diffusion has been discussed in detail elsewher $\mathrm{e}^{17}$ and found to make only a minor contribution to the overall transport processes. We neglect all contributions to diffusion arising from external forces and pressure gradients. These are usually of second order importance and can be neglected. We assume a three component system; acetic acid monomer, dimer, and inert carrier gas. This is reasonable since available thermodynamic data ${ }^{8-11}$ on acetic acid vapor suggests only a very minor contribution (if at all) from higher order associated clusters. We assume that the monomerdimer mixture can be considered to be everywhere in local equilibrium. The notion of local chemical equilibrium has been explored elsewhere $\mathrm{e}^{18-20}$ and found to be a good approximation for similar types of reactions, e.g., $2 \mathrm{NO}_{2} \rightleftharpoons \mathrm{N}_{2} \mathrm{O}_{4}$ and $6 \mathrm{HF}=(\mathrm{HF})_{6}$. Finally, we approximate the three component system as a perfect gas mixture. ${ }^{9-11}$

For the steady state, plane parallel diffusion condi- 
tions which prevail in the cloud chamber during normal operation, we write the continuity equations for each component as

$$
\begin{aligned}
& (d / d z)\left(l_{1}\right)=r_{1}, \\
& (d / d z)\left(l_{2}\right)=r_{2}, \\
& (d / d z)\left(l_{3}\right)=r_{3} .
\end{aligned}
$$

Here $l_{i}$ represents the ( $z$ direction) mass flux of the $i$ th species with respect to a stationary reference, and $r_{i}$ is the mass rate of change of species $i$. In our notation, the subscript 1 will represent monomer, the subscript 2 the dimer, and the subscript 3 the carrier gas. The conditions $r_{1}=-r_{2}$ and $r_{3}=0$ hold for our experiments. The mass flux of the $i$ th component can be expressed as a Stefan-Maxwell equation ${ }^{21}$ :

$$
\frac{d}{d z}\left(\frac{n_{i}}{n}\right)=\sum_{j \neq i} \frac{n_{i} n_{j}}{n^{2} D_{i j}}\left(\bar{V}_{j}-\bar{V}_{i}\right),
$$

where

$$
\bar{V}_{i}=\left(l_{i} / n_{i} m_{i}\right),
$$

$n_{i}$ is the molecular density of the $i$ th species, $n$ is the total molecular density, $D_{i j}$ is the binary diffusion coefficient, $\bar{V}_{i}$ is the diffusional velocity of the $i$ th species with respect to a stationary reference, and $m_{i}$ is the molecular mass of the $i$ th species.

It is important to realize that in our experiments we require the partial pressure of the acetic acid vapor, not the individual partial pressures of the monomer and dimer species. This also means that in our analysis we require the total mass flux and not the separate fluxes of the individual constituents. This is fortunate since, during steady operation, the total mass flux is constant throughout the chamber whereas the individual fluxes are not.

Writing Eq. (8) first for monomer and then for dimer and adding yields

$$
\frac{d}{d z}\left(\frac{n_{1}+n_{2}}{n}\right)=-\frac{n_{3} \beta}{n^{2} m_{1} D_{13}} l,
$$

where we have set $\bar{V}_{3}=0$ (carrier gas is stagnant) and used the fact that the binary diffusion coefficient is symmetrical, i. e., $D_{i j}=D_{j i}$. In Eq. (10) $l=l_{1}+l_{2}$ is the total mass flux (a constant) and

$$
\beta=(1-\phi)+\frac{m_{1} D_{13}}{m_{2} D_{23}} \phi,
$$

where $\phi=l_{2} / l$ is the dimer flux fraction. If we make the approximation that $m_{1} D_{13} / m_{2} D_{23} \cong \sqrt{2} / 2$, Eq. (11) may be written as

$$
\beta=1-0.29 \phi \text {. }
$$

The dimer flux fraction can be estimated using kinetic theory expressions for average velocities and equilibrium data for monomer and dimer concentrations. Equation (10) may be written more conveniently in terms of partial pressure and a reduced chamber height, $Z$, as

$$
\frac{d P}{d Z}=\frac{\left(P-P_{T}\right) \beta}{D_{13}^{0} T^{s}} L,
$$

where $P=P_{1}+P_{2}$ is the partial pressure of acetic acid, $P_{T}$ is the total pressure, $L$ is a reduced molar flux given by $(l h) /\left(M_{1}\right)$, where $M_{1}$ is the monomer molecular weight, and $h$ is the chamber height, and $Z=z / h$. The temperature and pressure dependence of the binary diffusion coefficient have been introduced explicitly ${ }^{22}$ with the relation

$$
D_{13}=\frac{D_{13}^{0} T^{s}}{C},
$$

where $C=P_{T} / R T$, and $D_{13}^{0}$, and $s$ are constants.

For the same one dimensional, three component system considered above, the energy flux equation assumes the form ${ }^{21}$

$$
q=-\lambda^{\prime}(d T / d z)+n_{1} h_{1} \bar{V}_{1}+n_{2} h_{2} \bar{V}_{2},
$$

where $q$ is the energy flux, $\lambda^{\prime}$ is the mixture thermal conductivity, and $h_{1}$ and $h_{2}$ represent the molecular enthalpys for monomer and dimer, respectively. The remaining symbols have been defined earlier. In writing Eq. (14) we again have made use of the fact that $\bar{V}_{3}=0$ and have neglected the effects of thermal diffusion. Using the total mass flux, $l=l_{1}+l_{2}$, and the reduced chamber height, we can rewrite Eq. (14) as

$$
Q=-\lambda(d T / d Z)+L H \text {. }
$$

Here $Q=h q$ is a reduced energy flux, $\lambda$ is the mixture thermal conductivity, $L$ is the same reduced mass flux defined above, and $H$ represents the molar enthalpy expression

$$
H=H_{1}+\phi\left(H_{2} / 2+H_{1}\right) .
$$

$\phi$ is the dimer flux fraction defined above and $H_{1}$ and $H_{2}$ are molar enthalpys for monomer and dimer, respectively.

Equations (13) and (15) are solved for the total acetic acid partial pressure and temperature profiles, respectively. Both of these equations are of the same form (except for the thermal diffusion terms) as earlier expressions for binary systems and are solved by similar procedures. ${ }^{13,14}$ Two important variations, however, involve the quantities $\phi$ and $\lambda$. The dimer flux fraction, $\phi$, was calculated assuming that the ratio of the steady concentration of monomer and dimer at any point in the chamber could be determined using equilibrium data. The value of $\phi$ generally varied from 0.7 to 0.9 in any one experiment. This means that a large fraction of the mass flux in any one experiment was due to dimer, and that the dimer contributed significantly to the pressure and temperature profiles in the chamber.

The second variation involves the mixture thermal conductivity, $\lambda$. The Wassiljewa equation and the Lindsay-Bromley correlation ${ }^{23}$ were used to calculate the mixture thermal conductivity from the conductivities of pure carrier gas, $\lambda_{c}$, and pure acetic acid vapor, $\lambda_{e}$. The acetic acid conductivity is of particular interest, however, since it is known ${ }^{24,25}$ that thermal conductivities in chemically reacting gas mixtures can be much greater than those in "frozen" gas mixtures of the same composition with no reaction. This in fact seems to be the case with acetic acid. Although experimental data is 


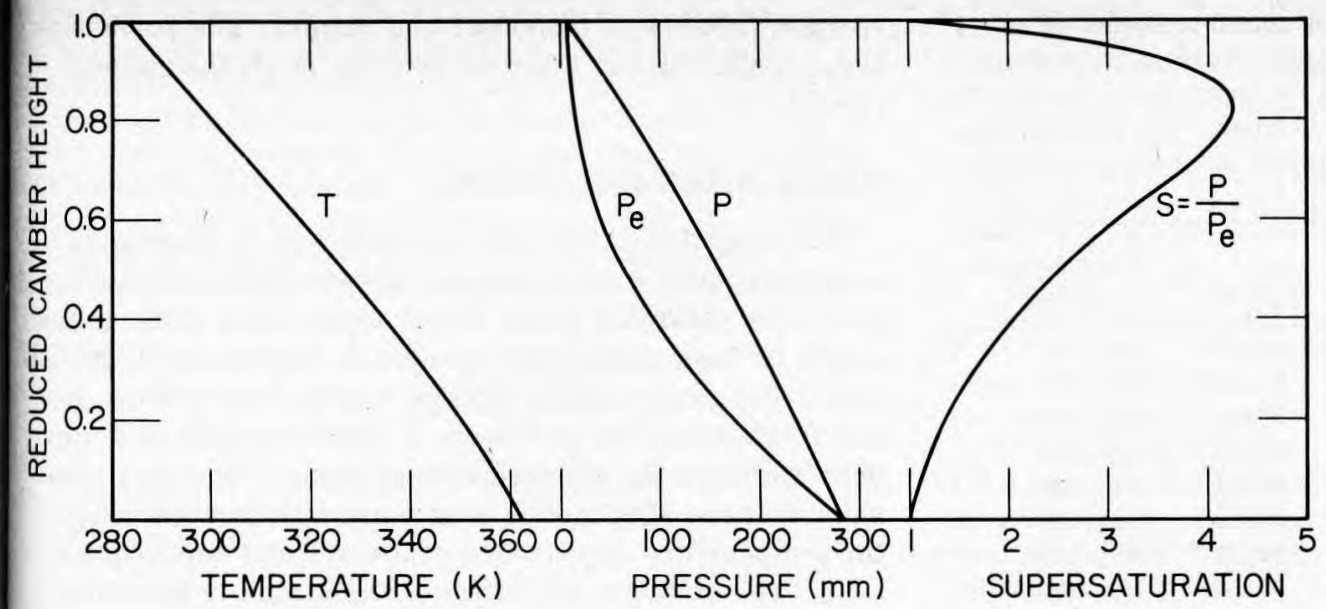

FIG. 2, Variation of temperature, saturation vapor pressure, partial pressure, and supersaturation as a function of reduced chamber height. The partial pressure and temperature curves represent solutions ot Eqs. (13) and (15), respectively, in the text. These plots represent actual data from experiment number 6 .

scarce, it clearly shows that the mixture conductivity is at least several times greater than that expected for a nonreacting gas mixture of monomer and dimer. ${ }^{25}$ To account for this an expression developed by Butler and Brokaw ${ }^{19,20}$ for the thermal conductivity in reacting vapor mixtures was used to calculate the pure acetic acid conductivity. Details of the calculation are given in the Appendix.

Typical solutions to Eqs. (13) and (15) are shown in Fig. 2. Here the curves represent actual data corresponding to experiment number 6 depicted in Fig. 3 .

\section{EXPERIMENTAL PROCEDURE}

Prior to an experiment the particular cloud chamber to be used was cleaned with soap and water, acetone, Micro cleaning solution, and finally acetic acid. Because acetic acid did not wet the Teflon, a thin layer of Pyrex glass wool was cleaned, dried, and applied to the upper plate surface of CC2. ${ }^{14}$ The glass wool was held in position by moistening with acetic acid. After assem- bly acetic acid was added to the chamber, and the chamber was then flushed several times with helium or hydrogen, the two carrier gases used in this investigation. The acetic acid used was J. T. Baker Ultrex brand, assayed at $99.8 \%$ purity. No additional purification was attempted. Both carrier gases were $99.99+\%$ purity. During operation thermocouple measurements were made with a Leeds and Northrop K-4 potentiometer in combination with a Keithley Model 155 microvolt null detector. A Houston Omniscribe chart recorder was used to display the thermocouple signals. A typical sequence for a given experiment is similar to that already described in detail in an earlier paper. ${ }^{14}$

In Table I experimental data consisting of the pool surface temperature $T_{0}$, the film surface temperature $T_{1}$, and total pressure $P_{T}$ are listed for each experiment. In Table II all the additional information necessary to compute partial pressure and temperature profiles for each experiment and the variation of the critical supersaturation with temperature for acetic acid from

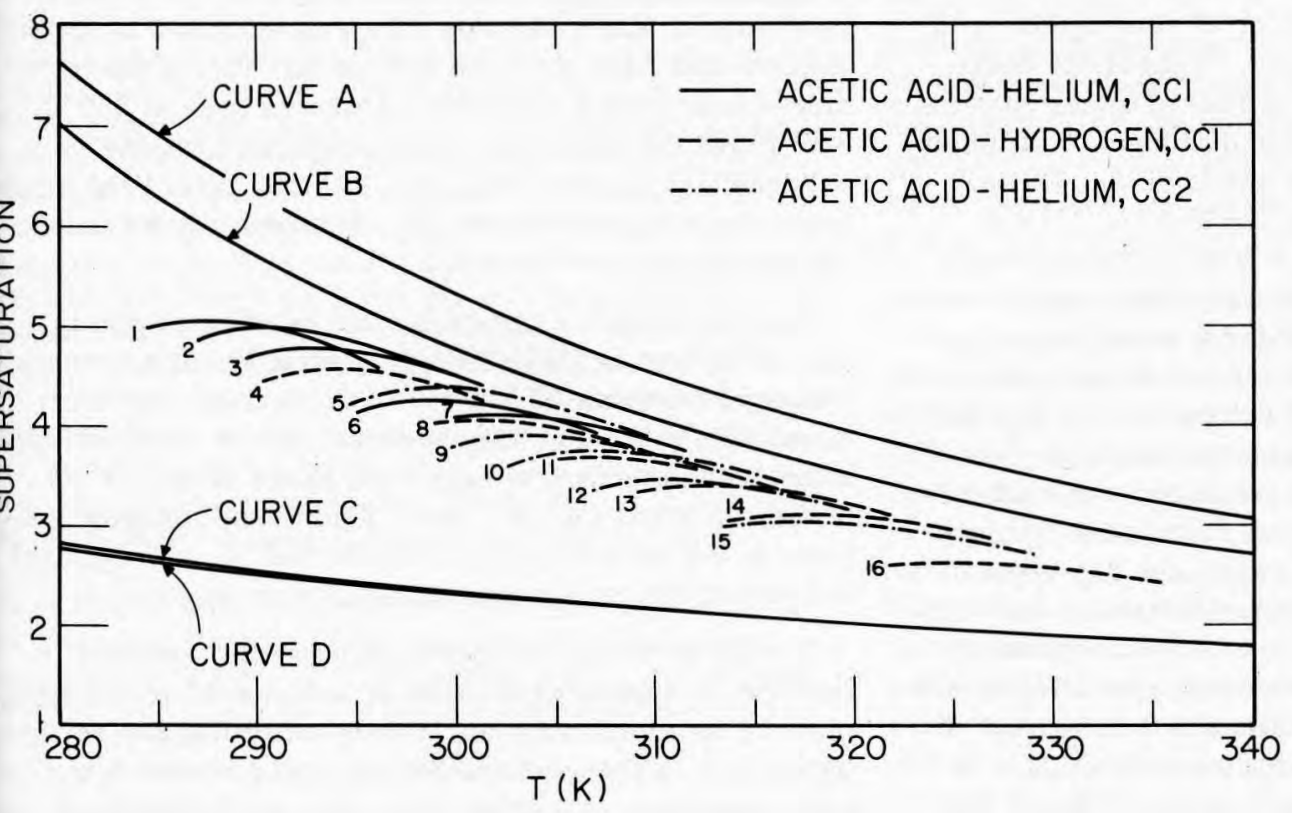

FIG. 3. The variation of the critical super saturation of acetic acid vapor as a function of temperature. Curves $\mathrm{C}$ and $\mathrm{A}$ represent the variation as predicted by the BeckerDöring theory for a vapor of pure monomer and of pure dimer acetic acid, respectively. Curves B and D are the variation for a monomerdimer mixture as predicted by the Katz, Saltsburg, Reiss theory and the Frisch, Willis theory, respectively. The envelope of the numbered curves is the experimentally measured variation. The solid curves denote experiments in $\mathrm{CCl}$ with helium as the carrier gas, the dashed curves denote experiments in $\mathrm{CCl}$ with hydrogen as the carrier gas, and the dot-dash curves denote experiments in CC2 with helium as the carrier gas. 
TABLE I. Experimental data for the acetic acid nucleation measurements: $T_{0}$ is the surface temperature of the working fluid, $T_{1}$ is the film temperature on the upper plate, and $P_{T}$ is the total pressure inside the cloud chamber. The carrier gas and particular cloud chamber used in any one experiment are noted in the last column.

\begin{tabular}{llrrl}
\hline \hline $\begin{array}{l}\text { Experiment } \\
\text { no. }\end{array}$ & $T_{0}\left({ }^{\circ} \mathrm{K}\right)$ & $T_{1}\left({ }^{\circ} \mathrm{K}\right)$ & $P_{T}(\mathrm{~mm})$ & $\begin{array}{l}\text { Carrier gas; } \\
\text { Chamber }\end{array}$ \\
\hline 1 & 351.5 & 273.5 & 693.5 & $\mathrm{He} ; \mathrm{CC} 1$ \\
2 & 355.5 & 276.1 & 913.0 & $\mathrm{He}$; CC1 \\
3 & 358.0 & 278.5 & 929.5 & $\mathrm{He}$ CCl \\
4 & 357.5 & 279.3 & 850.0 & $\mathrm{H}_{2} ; \mathrm{CC} 1$ \\
5 & 365.5 & 284.4 & 808.5 & $\mathrm{He} ; \mathrm{CC} 2$ \\
6 & 362.5 & 283.9 & 975.5 & $\mathrm{He} ; \mathrm{CC} 1$ \\
7 & 366.7 & 287.1 & 1007.5 & $\mathrm{He}$ CC1 \\
8 & 364.1 & 286.1 & 901.0 & $\mathrm{H}_{2} ; \mathrm{CC} 1$ \\
9 & 369.0 & 289.0 & 835.5 & $\mathrm{He}$ CC2 \\
10 & 376.1 & 292.5 & 850.2 & $\mathrm{He} ; \mathrm{CC} 2$ \\
11 & 369.3 & 291.6 & 945.5 & $\mathrm{H}_{2} ; \mathrm{CC} 1$ \\
12 & 378.9 & 295.6 & 880.0 & $\mathrm{He} ; \mathrm{CC} 2$ \\
13 & 374.4 & 296.9 & 1076.0 & $\mathrm{H}_{2} ; \mathrm{CC} 1$ \\
14 & 378.1 & 301.4 & 1121.0 & $\mathrm{H}_{2} ; \mathrm{CC} 1$ \\
15 & 383.1 & 301.4 & 1004.0 & $\mathrm{He}$ CC2 \\
16 & 383.1 & 309.0 & 1187.5 & $\mathrm{H}_{2} ; \mathrm{CC} 1$ \\
\hline \hline
\end{tabular}

Eqs. (1)-(3) are given. In keeping with convention the critical supersaturation is defined as that supersaturation which just supports a nucleation rate of $1 \mathrm{drop} / \mathrm{cm}^{3}$ $\mathrm{sec}^{-1}$. In Fig. 3 we present plots of the individual experiments. The envelope of these curves is the experimental value for the variation of critical supersaturation with temperature for acetic acid. The solid curves represent experiments in $\mathrm{CC} 1$ using helium as a carrier gas, the dashed curves represent experiments in CC1 using hydrogen, and the dot-dash curves represent experiments in CC2 using helium. The actual envelope has not been drawn for the sake of clarity.

Figure 3 also illustrates the predicted variation of the critical supersaturation for acetic acid as determined from the FW and KSR theories. Curves $\mathrm{C}$ and $\mathrm{A}$ were calculated from Eq. (2) for a vapor of pure acetic acid monomer and dimer, respectively. This is the variation of critical supersaturation predicted by the conventional nucleation theory, for a vapor composed of either pure monomer or of pure dimer acetic acid. These curves were obtained by setting $J^{\prime}=1$ in Eq. (2) and solving for the supersaturation at a given temperature. Curve B represents the variation in critical supersaturation predicted by the KSR theory for a vapor containing an equilibrium mixture of monomer and dimer acetic acid. This plot was obtained by setting $J=1$ in Eq. (3) and solving for $S$, the total supersaturation at a given temperature. In Eq. (3) $S_{1}$, the monomer supersaturation, is related simply to $S$ for our calculation because the monomer and dimer partial pressures are taken to be related through the equilibrium constant for the reaction in Eq. (4). Finally, curve D in Fig. 3 represents the variation in critical supersaturation predicted by the FW theory. This curve was obtained by setting $J_{\text {FW }}=1$ in Eq. (1) and again solving for the supersaturation at a given temperature. The surface fraction of dimer to monomer in Eq. (1) was calculated assuming an equi- librium mixture of monomer and dimer. The condensation coefficient was taken to be unity in all the calculations.

\section{RESULTS AND DISCUSSION}

The objective of this investigation was to determine experimentally if the presence of association clusters in a supersaturated vapor would render that vapor more stable or less stable with respect to homogeneous nucleation. The experimental data plotted in Fig. 3 show that, for acetic acid, the presence of large amounts of dimer does stabilize the supersaturated vapor. The data also show that the KSR theory does quite well in predicting the temperature dependence of the critical supersaturation. The stabilization effect of the dimer is dramatic. At $300^{\circ} \mathrm{K}$, for example, the experimental critical supersaturation (the envelope of the experimental curves) is roughly twice the critical supersaturation for the pure monomer calculation (curve $\mathrm{C}$ ). This corresponds to a decrease in the monomer nucleation rate (curve $C$ ) of nearly $10^{18}$. It should be remembered that there is a considerable amount of dimer present under these conditions; in fact, the dimer mole fraction at $300^{\circ} \mathrm{K}$ and a supersaturation of 4.5 is 0.91 .

The stabilizing effect of small, relatively stable clusters has been suggested previously for another quite different system. While examining the role of hydrates in the binary nucleation process in supersaturated sulfuric acid-water vapors, Heist and Reiss ${ }^{26}$ and later Shugard, Heist, and Reiss ${ }^{27}$ discovered that most of the sulfuric acid present in the vapor was hydrated and that these hydrates had the overall effect of dramatically reducing the binary nucleation rate.

We have, to this point, neglected the presence of any higher order association clusters in the acetic acid vapor. Although it has not been definitely established, Johnson and $\mathrm{Nash}^{9}$ suggested the existence of an acetic acid trimer and estimated the equilibrium constant for the monomer-trimer reaction. The effect of a trimer species upon the nucleation rate was examined using the Johnson and Nash data and solving Eq. (3) for the monomer-dimer-trimer system. This was done at $\mathbf{2 8 0}$ and $340^{\circ} \mathrm{K}$ and the resulting critical supersaturations were only slightly greater (less than $2 \%$ ) than that of the monomer-dimer system (curve B). Consequently we have neglected this contribution.

The equilibrium constant for the reaction shown in Eq. (4) (given in Table II) is only strictly valid for the temperature range 323 to $400^{\circ} \mathrm{K} .{ }^{11}$ It does, however, agree well with other experimental values when extrapolated over the entire temperature range $\left(298-473^{\circ} \mathrm{K}\right)$ of the published data. ${ }^{8,9}$ For calculations involving portions of the curves in Fig. 3 below $298^{\circ} \mathrm{K}$, extrapolated values for the equilibrium constant were used.

During the course of these experiments, we found it possible to supercool the film of acetic acid on the upper plate of the cloud chamber thereby extending our working range. Although supercooling was possible with both chambers, larger degrees of supercooling were possible with CC1. In one experiment we were able to 
TABLE II. Expressions for the acetic acid thermal conductivity, $\lambda_{e}$, carrier gas thermal conductivity, $\lambda_{c}$, saturation vapor pressure, $P_{e}$, surface tension, $\sigma$, vapor viscosity, $\eta$, density, $d$, monomer molar heat capacity at constant pressure, $C_{P_{1}}$, dimer molar heat capacity at constant pressure, $C_{P_{2}}$, and association equilibrium constant, $K_{12}$ and values for the monomer molecular weight, $M$, binary diffusion coefficient, $D_{13}$, and the binary diffusion coefficient temperature dependence, $s .^{2}$

Acetic acid:

$$
\begin{array}{r}
\lambda_{e}=\left(0.155+7.04 \times 10^{-7} T\right)\left[\frac{1.84 \times 10^{-5} T^{1.5}}{(960+T)}\right]+ \\
2.02 T^{-1.25}\left[\frac{X_{1} X_{2}}{\left(2 X_{2}+X_{1}\right)^{2}}\right]^{b}
\end{array}
$$

$$
\begin{aligned}
\ln P_{e} & =19.132-(4890 / T)^{\mathrm{c}} \\
\sigma & =29.427-0.0952(T-273.16)^{\mathrm{d}} \\
\eta & =177.9 \times 10^{-7} \frac{T^{1.5}}{(T+905.2)} \\
d & =1.0638-0.0012(T-273.16)^{\mathrm{f}} \\
C_{P_{1}} & =15.01+0.0356(T-273.16)^{\mathrm{g}} \\
C_{P_{2}} & =33.19+0.0720(T-273.16)^{\mathrm{g}} \\
\log _{10} K_{12} & =(3225 / T)-10.576^{\mathrm{h}}
\end{aligned}
$$

$M=60.05 ;{ }_{\mathrm{He}} D_{13}=0.3569^{\mathrm{i}} ;$

$$
\mathrm{H}_{2} D_{13}=0.4134^{1} ; s=0.75^{\mathrm{i}}
$$

Helium:

$$
\begin{aligned}
& \lambda_{c}=7.376974 \times 10^{-5}+1.139222 \times 10^{-6}-6.343536 \times 10^{-10} T^{2 j} \\
& \eta=145.5 \times 10^{-7} \frac{T^{1.5}}{(T+74.1)} \mathrm{k} \\
& M=4.0026
\end{aligned}
$$

Hydrogen:

$$
\begin{aligned}
\lambda_{c}=-5.468234 & \times 10^{-5} \\
& +2.137513 \times 10^{-6} T-1.697643 \times 10^{-9} T^{j} \\
\eta= & 64.9 \times 10^{-7} \frac{T^{1.5}}{(T+74)}{ }^{1} \\
M= & 2.0159
\end{aligned}
$$

${ }^{2} \lambda$ in cal $/\left(\mathrm{cm} \cdot \mathrm{sec} \cdot{ }^{\circ} \mathrm{K}\right), P_{e}$ in $\mathrm{mm}, \sigma$ in $\mathrm{erg} / \mathrm{cm}^{2}, \eta$ in $P$; $d$ in $\mathrm{g} / \mathrm{cm}^{3} ; C_{P}$ in $\left.\mathrm{cal} / \mathrm{mole} \cdot \mathrm{K}\right), K_{12}$ in $\mathrm{mm}^{-1} ; D_{13}$ in $\mathrm{cm}^{2} / \mathrm{sec}$ at $273.16^{\circ} \mathrm{K}$ and one atm, $s$ is dimensionless; $T$ in ${ }^{\circ} \mathrm{K}$.

${ }^{\mathrm{b}}$ The first term is the conductivity of pure monomer, $\lambda_{f}$, and the second term is the conductivity due to the chemical reaction, $\lambda_{R}$, (see Appendix). $\lambda_{f}$ was determined using the modified Eucken correction; high temperature viscosity data obtained from Perry's Chemical Engineers' Handbook, 4th ed. (McGraw-Hill, New York, 1963), pp. 3-197; and heat capacity data obtained from $\mathrm{W}$. Weltner, Footnote $\mathrm{g}$. $\lambda_{R}$ was determined by the method described in the Appendix.

The expression for the saturation vapor pressure was obtained from data of D. H. Stull, Ind. Eng. Chem. 39, 517 (1947); J. Timmermans, Physico-Chemical Constants of Pure Organic Compounds (Elsevier, New York, 1950), Vol. 1, p. 380; and the International Critical Tables (McGraw-Hill, New York, 1928), Vol. 3, p. 217.

The surface tension expression was obtained from data of J. Timmermans, Physico-Chemical Constants of Pure Organic Compounds (Elsevier, New York, 1950), Vol. 1, p. 382; and the Intemational Critical Tables (McGraw-Hill, New York, 1928), Vol. 4, p. 448.

"The expression for the vapor viscosity was obtained from data of A. G. Nasini, Phil. Mag. 8, 596 (1929); I. F. Golubev, Viscosity of Gases and Gas Mixtures (Israel Program for

\section{TABLE II (Continued)}

Scientific Transactions, Jerusalem, 1970), p. 66; and Perry's Chemical Engineers' Handbook, 5th ed. (McGraw-Hill, New

York, 1963), p. 3-211.

'Obtained from data in J. Timmermans, Physico-Chemical Constants of Pure Organic Compounds (Elsevier, New York, 1950), Vol. 1, p. 380.

${ }^{8}$ W. Weltner, J. Am. Chem. Soc. 77, 3941 (1955).

hJ. R. Barton and C. C. Hsu, J. Chem. Eng. Data 14, 184 (1969).

${ }^{1}$ E. N. Fuller, P. D. Schettler, and J. C. Giddings, Ind. Eng. Chem. 58, 19 (1966).

${ }^{j}$ Thermophysical Properties Research Center Data Book (Purdue University, 1970), Vol. 3.

${ }^{k}$ The expression for the viscosity of helium gas was obtained from the data of Onnes and Weber, Verk. K. Acad, Wet., Amsterdam, Afd. Natuurkd. 21, 1385 (1913) for the temperature range of $200-450{ }^{\circ} \mathrm{K}$.

${ }^{1}$ The expression for the viscosity of hydrogen gas was obtained using data from the International Critical Tables (McGrawHill, New York, 1929), Vol. 5, p. 5, for the temperature range $270-400^{\circ} \mathrm{K}$.

supercool the condensate film to $272^{\circ} \mathrm{K}$ before it froze. The normal freezing point of acetic acid is $289.8^{\circ} \mathrm{K} .{ }^{28}$ We found no information in the literature on thermodynamic properties of supercooled acetic acid. For the data in Fig. 3, at temperatures below the normal freezing point, we used extrapolated values for the properties calculated from the expressions given in Table II.

In all of our experiments we never observed an electric field effect on the nucleation rate. ${ }^{29}$ Normally at supersaturations somewhat lower than the critical supersaturation, nucleation occurs in the chamber. This nucleation is due to ions that are present and can be stopped by applying an electric field across the chamber to sweep out these charged particles. Using electric fields up to $140 \mathrm{~V} / \mathrm{cm}$, we were not able to visually discern any electric field effect upon the nucleation rate.

Finally, we make a few comments regarding the reliability of the experimental data in Fig. 3. First of all there was some question prior to our investigation concerning chemical attack of the aluminum plates in CC1 by the acetic acid. The data from the experiments with CC2 show this to be no problem. The results from CC1 and the Teflon coated CC2 are the same. In addition, there is no carrier gas dependence, the results are the same for hydrogen and for helium.

The validity of the solutions to the mass and energy transport equations for the diffusion cloud chamber have been discussed in much detail in the literature. ${ }^{13-15}$ Equations (13) and (15) are of the same general form and are solved by analogous techniques so that only a few additional points need be made here. The values for $D_{13}, s$, and $\lambda_{e}$ used in Eqs. (13) and (15) are probably known with the least certainty. Fortunately, the solutions are not critically dependent upon the values of these parameters. For instance, a variation of $\pm 25 \%$ in the value of $D_{13}$ will change the calculated supersaturation in any one solution by about $5 \%-6 \%$ and variations of $s$ of $\pm 33 \%$ will change the calculated supersaturation by only $1 \%-2 \%$. The solutions are somewhat less sensitive to the value of $\lambda_{e}$. Although the expression for $\lambda_{e}$ 
given in Table II reproduces published experimental conductivity data, usually to within a few percent, the published data is from a temperature range above that of our experiments. As a result the extrapolated values of $\lambda_{e}$ used in our calculations are somewhat uncertain. Fortunately, however, even a $\pm 50 \%$ variation in $\lambda_{B}$ only alters the calculated supersaturation by roughly $5 \%$.

\section{ACKNOWLEDGMENTS}

This research was supported by the National Science Foundation under Grants \#ENG75-15342 and \#GP28722X. Acknowledgment is made to the Donors of The Petroleum Research Fund, administered by the American Chemical Society, for the partial support of this research under Grant \#8296-G6, 7.

\section{APPENDIX}

The thermal conductivity of acetic acid vapor was obtained by a method due to Butler and Brokaw ${ }^{19,20}$ for calculating conductivities in chemically reacting vapor mixtures.

For a monomer-dimer mixture, assumed to be in local chemical equilibrium in the temperature gradient, the mixture conductivity is given by ${ }^{19}$

$$
\lambda_{e}=\lambda_{f}+\lambda_{R},
$$

where

$$
\lambda_{R}=\left(\frac{D_{12} P}{R T}\right)\left(\frac{\Delta H^{2}}{R T^{2}}\right) \frac{X_{1} X_{2}}{\left(2 X_{2}+X_{1}\right)^{2}} .
$$

Here $\lambda_{B}$ is the mixture thermal conductivity, $\lambda_{f}$ is the conductivity in the absence of chemical reaction, $\lambda_{R}$ is the conductivity due to the reaction, the subscripts 1 and 2 refer to monomer and dimer, respectively, $D_{12}$ is the monomer-dimer binary diffusion coefficient, $\Delta H$ is the enthalpy of the reaction shown in Eq. (4), $X_{i}$ is the mole fraction of the $i$ th species, and the remaining symbols have been defined in the text.

The $\Delta H$ term was evaluated using the Van't Hoff isochore; while the mole fractions were calculated using equilibrium data. $\lambda_{f}$ was taken to be the conductivity of pure monomer and calculated from high temperature vapor viscosity data using the modified Eucken correction. As experimental values for $D_{12}$ were not available, the value giving the best agreement with available conductivity data ${ }^{25}$ was used. This expression is

$$
D_{12}=1.51 \times 10^{-6}\left(T^{1.75} / P\right) \text {. }
$$

The expression for the conductivity due to the chemical reaction is

$$
\lambda_{R}=2.02 T^{-1.25} \frac{X_{1} X_{2}}{\left(2 X_{2}+X_{1}\right)^{2}}
$$

*To whom all correspondence should be addressed.

† Present address: Mobil Chemical Co., 100 North Street, Canandaigua, New York 14424.

${ }^{1}$ M. Volmer and A. Weber, Z. Physik. Chem. (Leipzig) 119, 277 (1925).

${ }^{2}$ R. Becker and W. Döring, Ann. Physik 24, 719 (1935).

${ }^{3}$ J. Frenkel, Kinetic Theory of Liquids (Oxford University, London, 1946), Chap. 7.

${ }^{4}$ J. Zeldovich, J. Expt. Theor. Phys. (Russ.) 12, 525 (1942).

${ }^{5}$ H. L. Frisch and C. Willis, J. Chem. Phys. 22, 243 (1954).

${ }^{6}$ J. L. Katz, H. Saltsburg, and H. Reiss, J. Colloid Interface Sci. 21, 560 (1966).

${ }^{7}$ C. S. Kiang, D. Stauffer, G. H. Walker, O. P. Puri, J. D. Wise Jr., E. M. Patterson, J. Atmos. Sci. 28, 1222 (1971). ${ }^{8}$ F. H. MacDougall, J. Am. Chem. Soc. 58, 2585 (1936).

${ }^{9}$ E. W. Johnson and L. K. Nash, J. Am. Chem. Soc. 72, 547 (1950).

${ }^{10} \mathrm{~W}$. Weltner, J. Am. Chem. Soc. 77, 3941 (1955).

${ }^{11}$ J. R. Barton and C. C. Hsu, J. Chem. Eng. Data 14, 184 (1969).

${ }^{12}$ J. L. Katz and B. J. Ostermier, J. Chem. Phys. 47, 478 (1967).

${ }^{13}$ J. L. Katz, J. Chem. Phys. 52, 4733 (1970).

${ }^{14}$ R. H. Heist and H. Reiss, J. Chem. Phys. 59, 665 (1973).

${ }^{15}$ J. L. Katz, .C. J. Scoppa II, N. G. Kumar, and P. Mirabel, J. Chem. Phys. 62, 448 (1975).

${ }^{16} \mathrm{H}$. Reiss and D. Marvin, private communication.

${ }^{17}$ I. Prigogine and R. Buess, Acad. R. Belg. Cl. Sci. Mem. Collect. 38, 711, 851 (1952).

${ }^{18}$ J. O. Hir schfelder, J. Chem. Phys. 26, 274 (1957).

${ }^{19}$ J. N. Butler and R. S. Brokaw, J. Chem. Phys. 26, 1636 (1957).

${ }^{20}$ R. S. Brokaw, J. Chem. Phys. 35, 1569 (1961).

${ }^{21}$ R. B. Bird, W. E. Stewart, and E. N. Lightfoot, Transport Phenomena (Wiley, New York, 1960), Chap. 18.

${ }^{22}$ E. N. Fuller, P. D. Schettler, and J. C. Giddings, Ind. Eng. Chem. 58, 19 (1966).

${ }^{23}$ R. C. Reid and T. K. Sherwood, The Properties of Gases and Liquids, 2nd ed. (McGraw-Hill, New York, 1966), Chap. 10.

${ }^{34}$ W. Nernst, Boltzmann-Festschrift 904 (1904).

${ }^{25}$ A. A. Tarzimanov and V. E. Mashirov, Thermophysical Properties of Matter and Substances, ed. by. V. A. Rabinovich, (Amerind, New Delhi, 1974), Vol. 2, Chap. 3.

${ }^{26}$ R. H. Heist and H. Reiss, J. Chem. Phys. 61, (2), 573 (1974).

${ }^{27}$ W. J. Shugard, R. H. Heist, and H. Reiss, J. Chem. Phys. 61, 5298 (1974).

${ }^{28} \mathrm{~J}$. Timmermans, Physico-Chemical Constants of Pure Organic Compounds (El sevier, Amsterdam, 1950), Vol. 1, p. 381 .

${ }^{29}$ T. H. Laby, Phil. Trans. R. Soc. London Ser. A 208, 445 (1908). 